\title{
First case report of Cohen syndrome in the Tunisian population caused by VPS13B mutations
}

\author{
Imen Rejeb ${ }^{1 *}$ (D), Houweyda Jilani ${ }^{1}$, Yasmina Elaribi', Syrine Hizem', Lamia Hila², Julia Lauer Zillahrdt ${ }^{3,4,5}$,
} Jamel Chelly ${ }^{3,4,5}$ and Lamia Benjemaa ${ }^{1}$

\begin{abstract}
Background: Cohen syndrome is a rare autosomal recessive developmental disorder that comprises variable clinical features counting developmental delay, pigmentary retinopathy, myopia, acquired microcephaly, truncal obesity, joint hypermobility, friendly disposition and intermittent neutropenia. VPS13B (vacuolar protein sorting 13, yeast, homologue of B) gene is the only gene responsible for Cohen Syndrome, causative mutations include nonsense, missense, indel and splice-site variants. The integrity of the Golgi apparatus requires the presence of the peripheral membrane protein VPS13B that have an essential function in intracellular protein transport and vesicle-mediated sorting.

Case presentation: In this study, we performed whole exome sequencing (WES) in a Tunisian family with two young cases having developmental delay, hypotonia, autism spectrum disorder, ptosis and thick hair and eyebrows. The proposita presented also pigmentory retinopathy. Compound heterozygous mutation in VPS13B gene was detected by WES. This mutation inherited from healthy heterozygous parents, supports an unpredictable clinical diagnosis of Cohen Syndrome. The proband's phenotype is explained by the presence of compound heterozygous mutations in the VPS13B gene. This finding refined the understanding of genotype-phenotype correlation.
\end{abstract}

Conclusions: This is the first report of a Tunisian family with Cohen syndrome mutated in the VPS13B gene.

Keywords: Cohen syndrome, VPS13B gene, Compound heterozygous mutation

\section{Background}

Cohen syndrome (CS) (MIM\# 216550) is a rare autosomal recessive developmental disorder characterized by Cohen and colleagues in 1973 [1]. Truncal obesity, intellectual disability, developmental delay, joint laxity, craniofacial dysmorphism, high myopia and/or retinal dystrophy and neutropenia are typical clinical manifestations of the syndrome $[1,2]$.At present, CS has been essentially assigned to mutations in the VPS13B gene (MIM\# 607817) among patients from diverse ethnicity. VPS13B, the single CS linked gene so far described, is localized on q22.2 locus of chromosome 8 . Its length is about $864 \mathrm{~kb}$ and comprises 62 exons. The longest transcript [NM_017890.4] is 14,100 bp long encoding for a 4022 amino acid protein.

\footnotetext{
* Correspondence: imen_rejeb@yahoo.fr; imen.rejeb@rns.tn

${ }^{1}$ Service des Maladies Congénitales et Héréditaires, CHU Mongi Slim La

Marsa, Sidi Daoud La Marsa, 2046 Tunis, Tunisia

Full list of author information is available at the end of the article
}

$V P S 13 B$ is a peripheral membrane protein with putative transmembrane domains and functional motifs that have an essential function in the transport of intracellular proteins and in vesicle-mediated sorting [3]. The expression of the VPS13B is mainly noticed in the whole body and in the central nervous system, blood, muscles, and heart [4]. Approximately, 200 cases of the CS and about more than 150 deleterious mutations have been identified to date (http://www.hgmd.org); in most cases mutations are stop codon mutations that result in a functionally null protein. The diagnosis is always difficult in childhood, this is due to the fact that many of the typical traits may be nonexistent till scholarisation or upcoming years and intermittent neutropenia is not consistently observable.

Here we report the characterization of a new compound heterozygous mutation in VPS13B gene in 2 Tunisian related cases with CS. 


\section{Case presentation}

The present study describes clinical and molecular findings in two patients with CS from a non-consanguineous Tunisian family. One of the authors examined the patients. DNA was extracted from peripheral blood using standard methods. The patients' parents gave their consent.

The proposita was the first child of non-consanguineous and healthy condition parents. Pregnancy was normal and carried to term with an APGAR score of 9 and 10 at one and five minutes after birth, respectively. The parameters at birth were: weight $3300 \mathrm{~g}$ (60th percentile), height $50 \mathrm{~cm}$ (70 th percentile), occipitofrontal circumference (OFC) $35 \mathrm{~cm}$ (85th percentile). Hypotonia and poor sucking were noticed. She presented delayed psychomotor development: she wasn't capable to sit without help till 12 months and walked at the age of 2 years. At 3 years, the patient was not capable to speak. Ophthalmological evaluation showed left strabismus and pigmentary retinopathy.

When reevaluated at 12 years, growth retardation and progressive microcephaly were noted. We noticed a weight of $31 \mathrm{~kg}(-1 \mathrm{SD})$, a height of $126 \mathrm{~cm}(-3.2 \mathrm{SD})$ and an OFC of $48 \mathrm{~cm}(-3.8 \mathrm{SD})$. At neurological assessment, we noticed widespread hypotonia and joint hypermobility. Clinical diagnosis revealed dysmorphic facial features as thick hair eye brows and lashes, prominent upper central incisors, prominent lips and short philtrum; the last two features lead to a half open-mouth. The hands were small with tapering fingers. She also presented truncal obesity (Fig. 1). Communication and social skills were impaired. She presented intellectual disability with autistic-like traits.

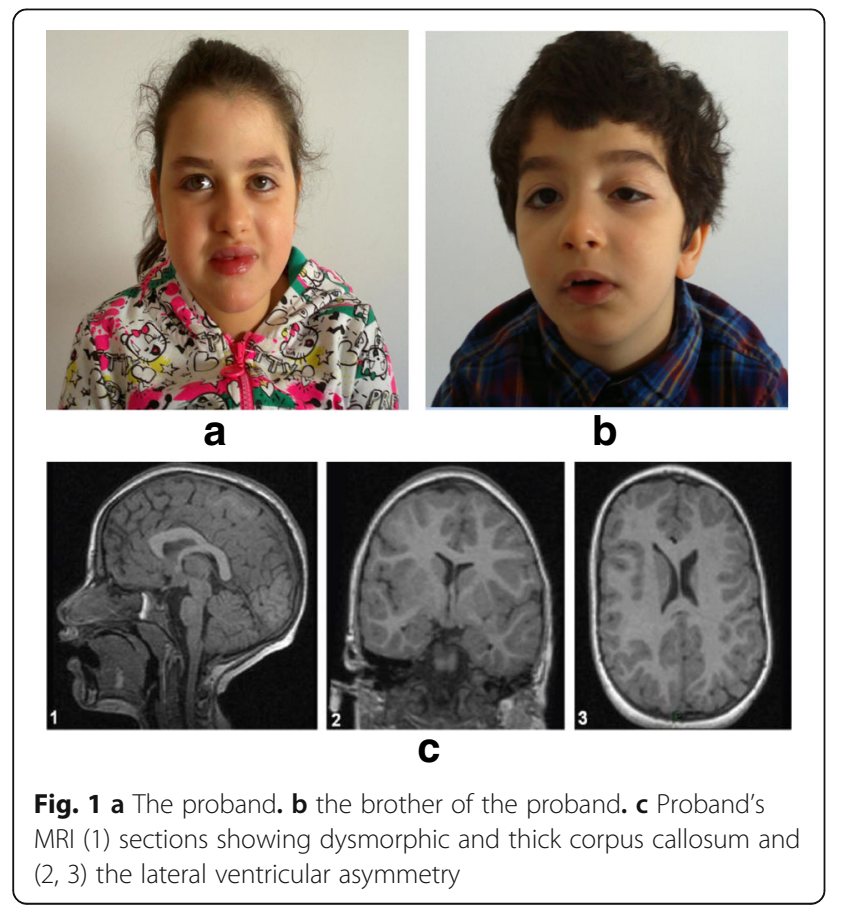

Cerebral MRI showed thick and dysmorphic corpus callosum and lateral ventricular asymmetry. The brainstem and the cerebellum were normal (Fig. 1c). Karyotype and $\mathrm{CGH}$ array were normal.

Her brother presented at the age of 6 years a weight of $22 \mathrm{~kg}$ (normal), a height of $113 \mathrm{~cm}$ (normal) and an OFC of $48 \mathrm{~cm}(-3 \mathrm{SD})$. Birth parameters were: weight $3350 \mathrm{~g}$ (50th percentile), height $50 \mathrm{~cm}$ (50th percentile), occipitofrontal circumference (OFC) $36 \mathrm{~cm}$ (85th percentile). Pregnancy was normal and carried to term with an APGAR score of 10 and 10 at one and five minutes after birth, respectively. $\mathrm{He}$ was born with a ptosis in the left eye operated at the age of 5 years (Fig. 1). He presented high myopia. He had, like his sister, thick hair eyebrows and lashes, he presented downslanted palpebral fissures, micrognathia, arched palate, clinodactyly of the toes, slender hands and feet and tapering fingers. Intellectual disability and stereotyped motor behavior were also noticed.

After the identification of the causal mutation, the reverse phenotyping showed moderate neutropenia and mild neutropenia in the proposita and her brother respectively. In fact, neutropenia is one of the important clinical signs of CS, but because of the nonexistence of clinical signs and the occasional occurrence, it is rarely identified.

The CARE guidelines were followed.

\section{Genetic testing}

Because of limitations of renseignement on neutropenia and pigmentary retinopathy we didn't notice that the clinical features matched with a CS, so we first use WES approach. We performed WES in trio made of the proband and his two unrelated parents.

Library generation, exome enrichment and whole-exome sequencing methodology used are detailed in the article written by Poirier et al. [5].

We analyzed variants affecting coding regions and essential splicing sites and excluded all variants with a frequency greater than $1 \%$ according to genomic databases (dbSNP, 1000 Genomes, Exome variant server and local platform database). All relevant variant were visually explored with Integrative Genomics Viewer (IGV: http://software.broadinstitute.org/software/igv/) to detect false positive results.

With this method and these filters, 9 variants were detected in index case (7 with de novo model of inheritance and 2 in the same gene with recessive model). 5 out of the 7 "de novo" variants appeared inherited from one parent (IGV), the 2 others were absent in the affected brother. The 2 variations in VPS13B gene (identified by recessive model of analysis) were also present in the brother; the c.3582delT, p.A1194fs were inherited from the mother and the c.6295_6296delAT, p.M2124 fs one from father.

Finally, we confirmed by PCR and Sanger sequencing of all coding regions and exon-intron boundaries of the VPS13B gene the relevant variants identified by WES. Using genomic 
DNA from the proband, her parents and her brother, the mutations identified were tested for familial segregation.

WES of individual II1 noticed the presence of a compound heterozygous variant $(100,479,778 \mathrm{~T} /-, 100712001 \mathrm{AT} /-)$ in the VPS13B gene (Fig. 2a). This compound heterozygous mutation in the VPS13B gene (NM_017890.4) was validated by Sanger sequencing (c.3582delT, p.A1149fs + c.6295_6 296delAT, p.M2124 fs) (Fig. 2b) inherited from healthy heterozygous parents confirms the diagnosis of CS.

Sanger sequencing showed that the variations c.3582delT (p.A1149fs) and c.6295_6296delAT (p.M2124 fs) in the VPS13B gene present in both the proband and her brother were of biparental origin (Fig. 3).

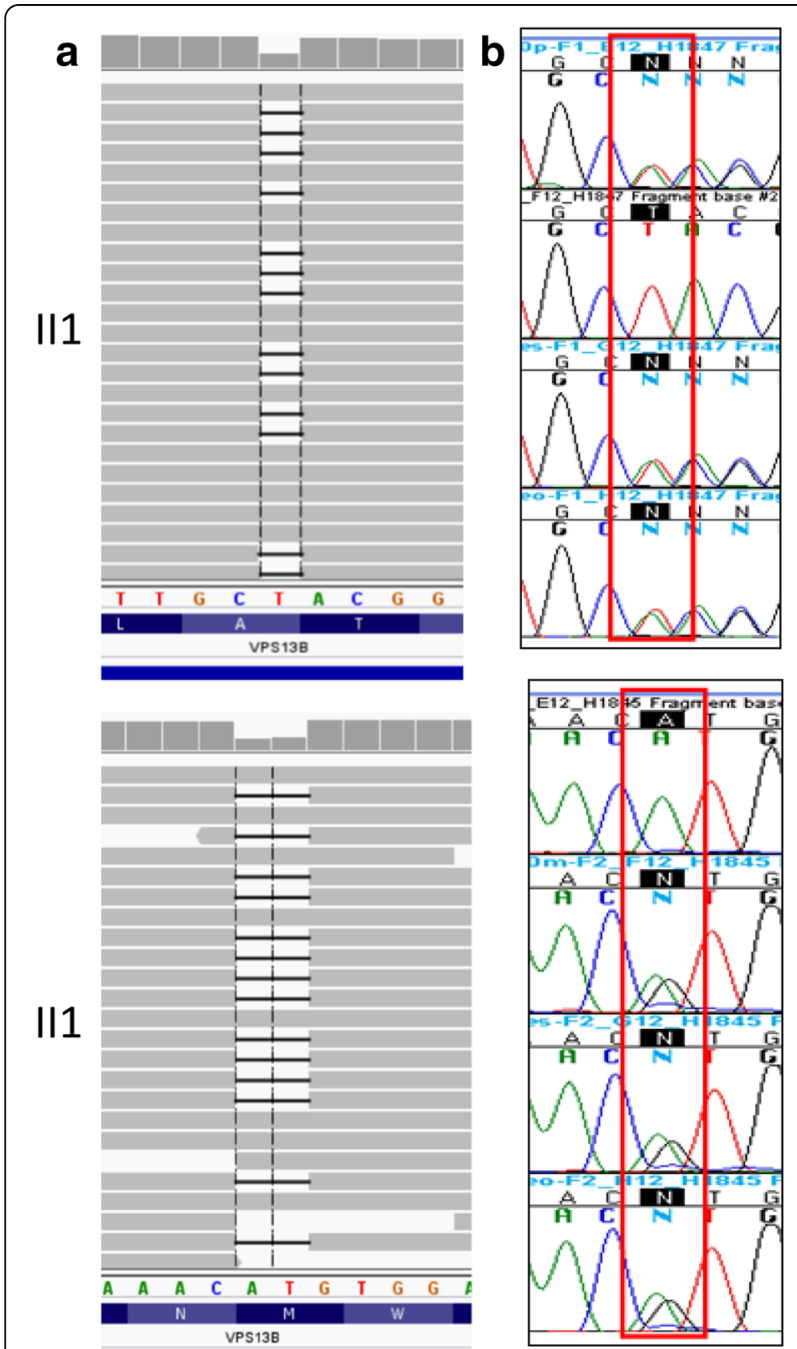

Fig. 2 a Integrative Genomics Viewer of short read alignment indicated the compound heterozygous variant identified by exome sequencing $(100,479,778 \mathrm{~T} /-, 100712001 \mathrm{AT} /-)$ in the VPS13B gene. b Sanger validation shows the compound heterozygosity of the proband $\| 1$ formed by the c.3582delT mutation, and the c.6295_6296delAT mutation

\section{Discussion and conclusions}

We report a Tunisian family including two siblings with developmental delay and intellectual disability harbouring a novel compound heterozygous mutation in the VPS13B gene by WES. Even for an experienced clinician, the diagnosis of CS is difficult. In fact this syndrome is a rare autosomal recessive disorder and it is often impossible to diagnosis CS until middle or late childhood. Therefore the phentotypic traits are very variable, several could be lacking till scholarisation or upcoming years. CS can be retained when six of eight phentotypic traits are noticed including developmental delay, joint hypermobility, typical CS facial gestalt, high myopia and/or retinal dystrophy, microcephaly, truncal obesity with slender extremities, overly sociable behaviour and neutropenia [6]. However some of these features cannot be observable till scholarisation or upcoming years, like retinal dystrophy, truncal obesity and overly sociable behavior, furthermore the typical facial gestalt of prominent incisors is always absent. In fact, when the girl was first seen at 7 years and the boy at 1 year, respectively, they had postnatal-onset microcephaly and delayed developmental milestones, we didn't suspect the CS at this time given no other revealing traits.

Mutations in the VPS13B gene are responsible for CS. The novel compound heterozygous mutation in VPS13B inherited from healthy heterozygous parents, c.3582delT (p.A1149fs) and c.6295_6296delAT (p.M2124 fs) inherited respectively from the father and the mother are present in the proband and her brother causes a frameshift that induce a premature stop codon. Subsequently this frameshift mutation generate a premature stop codon that can either induce a truncated protein lacking a number of functional domains of the VPS13B protein or to a functional null-allele as a result of a non sense mediated mRNA decay (NMD).

Actually, approximately 188 mutations in VPS13B gene have been identified, 153 of them were associated with CS (http://www.hgmd.org/). VPS13B is a Golgi-associated peripheral membrane protein co-localizing with the cis-Golgi matrix protein GM130. RP2 and RPGR, two retinitis pigmentosa disease genes, situate to the Golgi, and depletion of RP2 causes abnormal Golgi function and protein transport in the photoreceptor $[7,8]$. This highlights that a normal function of Golgi-associated proteins, including VPS13B, is essential for a good functioning of the photoreceptor. Recent studies have concluded that VPS13B mutations, responsible for $\mathrm{COH} 1$, are responsible of a tissue-specific major defect of glycosylation and endosomal-lysosomal trafficking defect. This highlights that VPS13B is essential in Golgi glycosylation and morphology, as well as in lysosomal-endosomal pathway maintenance [9].

Using WES in the early stage of disease for young patients where the clinical diagnosis is not evident is actually the best approach. Currently, the exome sequencing approaches are used in many laboratories helping 

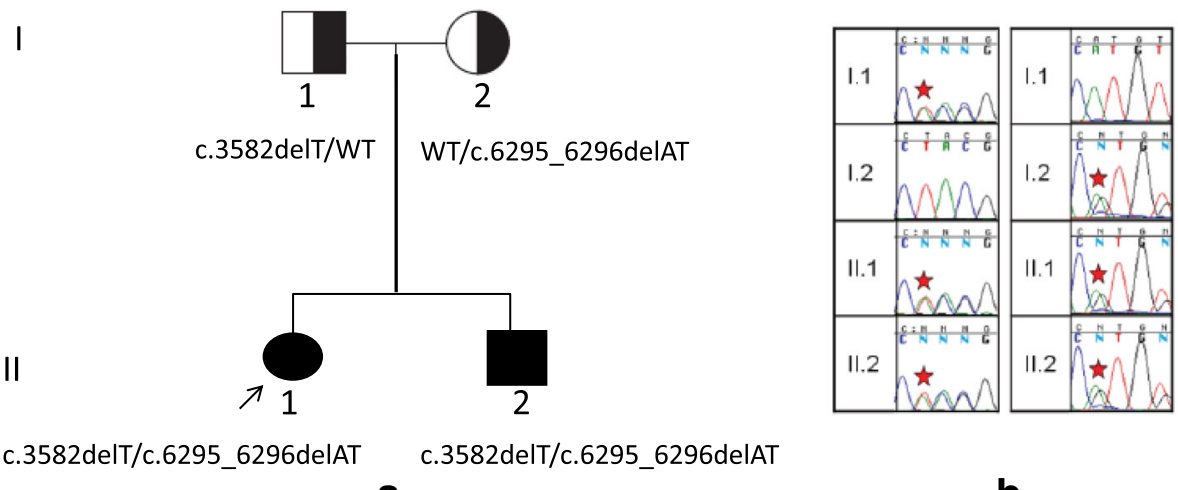

a

b

\section{Cohen syndrome}

Fig. 3 a Pedigree of the Tunisian family and compound heterozygosity of the proband, formed by the c.3582delT mutation, inherited from the father of the proband and also present in her brother, and the c.6295_6296delAT mutation inherited from the mother. The 2 mutations are shown in black, and in white the wild type alleles (WT). b Sanger validation shows segregation of the VPS13B mutations in the four family members

clinicians in solving the aetiology of many rare diseases. This approach, compared to sanger sequencing helps saving costs and time especially for large genes such as VPS13B gene. In fact, the costs of sequencing per base with sanger sequencing is much higher than with NGS [10, 11].So, sanger sequencing will be performed only if a causative mutation will be identified by NGS in order to validate this variant. Therefore, for mendelian diseases, especially for those with genetic heterogeneity, NGS is almost certainly the best primary choice in genetic tests.

In conclusion, we report the first Tunisian family with CS, a novel compound heterozygous mutation in VPS13B gene, identified using WES is the deleterious mutation in the patients of this family.

\section{Abbreviations}

APGAR: Appearance pulse grimace activity and respiration; CGH: Comparative genomic hybridization; CS: Cohen syndrome; MRI: Magnetic resonance imaging; NGS: Next-Generation Sequencing; NMD: Nonsense-mediated mRNA decay; OFC: Occipitofrontal circumference; PCR: Polymerase chain reaction; WES: Whole exome sequencing

\section{Acknowledgments}

We would like to thank the family members for their invaluable cooperation and participation.

\section{Funding}

Not applicable.

\section{Availability of data and materials}

All data generated or analyzed during this study are included in this published article.

\section{Authors' contribution}

LB and JC designed and initiated the study, monitored data collection and analysis for the study and revised the paper. IR contributed to sample collection, analyzed clinical data, and drafted the paper. HJ, YE and SH contributed to collection of clinical and imaging Data and genetic counseling. JLZ analyzed the WES data and revised the paper. LH helped with technical parts and revised the paper. All authors read and approved the final manuscript.
Ethics approval and consent to participate

This study was approved by the Ethics Review Committee CHU Mongi Slim La Marsa in Tunisia and informed consent was obtained from the patients' parents prior to participation.

\section{Consent for publication}

Consent for publication of respective case presentations was obtained from patients' parents. They give their consent for the publication of the medical data and photos of their son and daughter.

\section{Competing interests}

The authors declare that they have no competing interests.

\section{Publisher's Note}

Springer Nature remains neutral with regard to jurisdictional claims in published maps and institutional affiliations.

\section{Author details}

${ }^{1}$ Service des Maladies Congénitales et Héréditaires, CHU Mongi Slim La Marsa, Sidi Daoud La Marsa, 2046 Tunis, Tunisia. ${ }^{2}$ Laboratoire de Génétique Humaine, Faculté de Médecine de Tunis, Tunis, Tunisia. ${ }^{3}$ Institut Cochin, Université Paris-Descartes, CNRS (UMR 8104), Paris, France. ${ }^{4}$ Inserm, U1016, Paris, France. ${ }^{5}$ Pôle de biologie, Hôpitaux Universitaires de Strasbourg, Strasbourg, France.

Received: 24 March 2017 Accepted: 7 November 2017

Published online: 17 November 2017

\section{References}

1. Cohen MM Jr, Hall BD, Smith DW, Graham CB, Lampert KJA. New syndrome with hypotonia, obesity, mental deficiency and facial, oral, ocular and limb anomalies. J Pediatr. 1973;83:280-4.

2. Norio R, Raitta C, Lindahl E. Further delineation of the Cohen syndrome; report on chorioretinal dystrophy, leukopenia and consanguinity. Clin Genet. 1984;25:1-14.

3. Seifert W, Kühnisch J, Maritzen T, Horn D, Haucke V, Hennies HC. Cohen syndromeassociated protein, $\mathrm{COH} 1$, is a novel, giant Golgi matrix protein required for Golgi integrity. J Biol Chem. 2011;286:37665-75.

4. Seifert W, Holder-Espinasse M, Kühnisch J, Kahrizi K, Tzschach A, et al. Expanded mutational spectrum in Cohen syndrome, tissue expression, and transcript variants of $\mathrm{COH}$. Hum Mut. 2009:30:E404-20.

5. Poirier K, Lebrun N, Broix L, et al. Mutations in TUBG1, DYNC1H1, KIF5C and KIF2A cause malformations of cortical development and microcephaly. Nat Genet. 2013;45:639-47.

6. Kolehmainen J, Black GC, Saarinen A, et al. Cohen syndrome is caused by mutations in a novel gene, $\mathrm{COH} 1$, encoding a transmembrane protein with 
a presumed role in vesicle-mediated sorting and intracellular protein transport. Am J Hum Genet. 2003;72:1359-69.

7. Evans RJ, Schwarz N, Nagel-Wolfrum K, Wolfrum U, Hardcastle AJ, Cheetham ME. The retinitis pigmentosa protein RP2 links pericentriolar vesicle transport between the Golgi and the primary cilium. Hum Mol Genet. 2010; 19:1358-67.

8. Yan D, Swain PK, Breuer D, et al. Biochemical characterization and subcellular localization of the mouse retinitis pigmentosa GTPase regulator (mRpgr). J Biol Chem. 1998;273:19656-63.

9. Duplomb L, Duvet S, Picot D, et al. Cohen syndrome is associated with major glycosylation defects. Hum Mol Genet. 2014;23(9):2391-9.

10. Metzker ML. Sequencing technologies the next generation. Nat Rev Genet. 2010;11:31-46

11. Liu L, Li Y, Li S, et al. Comparison of next generation sequencing systems. J Biomed Biotechnol. 2012;2012:251364.

Submit your next manuscript to BioMed Central and we will help you at every step:

- We accept pre-submission inquiries

- Our selector tool helps you to find the most relevant journal

- We provide round the clock customer support

- Convenient online submission

- Thorough peer review

- Inclusion in PubMed and all major indexing services

- Maximum visibility for your research

Submit your manuscript at www.biomedcentral.com/submit
Biomed Central 